\title{
Global Research on COVID-19 Disease: A Scientific Assessment of Publications during 2020-21
}

\author{
BM Gupta ${ }^{1, *}$, SM Dhawan ${ }^{2}$, KK Mueen Ahmed ${ }^{3}$, Ghouse Modin Mamdapur ${ }^{4}$
}

\section{BM Gupta ${ }^{1}, *$ SM Dhawan², KK Mueen Ahmed', Ghouse Modin Mamdapur ${ }^{4}$ \\ 'Formerly with CSIR-NISTADS, New Delhi, INDIA. \\ ${ }^{2}$ Formerly with CSIR-NPL, New Delhi, INDIA. \\ ${ }^{3}$ Phcog.Net, Bengaluru, Karnataka, INDIA. \\ ${ }^{4}$ Synthite Industries $(P) L t d$, Kolenchery, Kerala, INDIA.}

\author{
Correspondence \\ Dr. BM Gupta, \\ Formerly with CSIR-NISTADS, New Delhi \\ 11012, INDIA \\ Mobile no: +919888378275 \\ Email: bmgupta1@gmail.com \\ History \\ - Submission Date: 07-01-2021; \\ - Revised Date: 02-02-2021; \\ - Accepted Date: 28-02-2021;
}

DOI : 10.5530/ijmedph.2021.2.14

Article Available online

http://www.ijmedph.org/v11/i2

\section{Copyright}

(C) 2021 Phcog.Net. This is an openaccess article distributed under the terms of the Creative Commons Attribution 4.0 International license.

\begin{abstract}
The study aims to evaluate global research output (103054 records) on coronavirus disease (COVID-19) based on quantitative and qualitative indicators. It presents a bibliometric profile of most influential countries, organizations, authors and journals and their collaborative linkages, besides identifying broad subject areas of research, most significant keywords and highly-cited papers related to COVID-19. During 2020-21, more than 150 countries participated in COVID-19 research, of which top 10 countries accounted for a $82.93 \%$ global share. The USA, China and U.K. produced the most articles $(25.86 \%, 10.77 \%$ and $10.7 \%$ respectively). The top three organizations, namely Harvard Medical School, USA, Huazhong University of S\&T, China and Tongji Medical College, China contributed 1755, 1344 and 1267 papers, respectively. The most cited organizations in COVID-19 research are: Peking Union Medical College, China (97.41 CPP), University of Hong Kong (82.17 CPP) and Renmin Hospital of Wuhan University, China (59.88 CPP). The top three most productive authors are: V. Wanitlit, E. Mahase and G. Lacobucci (with 152, 135 and 108 papers). The top three most cited authors in terms of citations per paper (CPP) are: Y. Hu (319.82), L. Liu (303.91) and J.F.W. Chan (169.55). The most productive organizations originate from most productive countries and they contribute the most collaborative papers. The top three journals, namely International Journal of Environmental Research, BMJ and PLOS One contributed 961, 854 and 781 papers, respectively. The top three journals in terms of citations per paper (CPP) are New England Journal of Medicine (122.78), The Lancet (106.3) and JAMA (75.17). The most ubiquitous topic was COVID-19 clinical studies, with maximum focus on virological aspects $(9.58 \%)$, respiratory aspects $(6.97 \%)$, molecular aspects $(3.11 \%)$, genetic aspects (3.09\%) and immunological aspects (1.3\%). The results of this study should be of interest to working scientists, clinicians and policy-makers in improving their understanding of COVID-19 disease and in its management.

Key words: COVID-19, Coronavirus Virus Disease 2019, SARS-CoV2, Global Publications, Bibliometrics, Scientometrics.
\end{abstract}

\section{INTRODUCTION}

The Coronavirus Virus Disease 2019 (COVID-19) is a contagious disease caused by severe acute respiratory syndrome coronavirus 2 (SARS-CoV-2). WHO first learned of this new virus on 31 December 2019, following a report of a cluster of cases of 'viral pneumonia' in Wuhan, China. The disease has since spread world-wide, leading to an ongoing pandemic. The COVID-19 aggressively spread throughout China in the following several weeks and became a worldwide pandemic affecting almost every country in the world within the subsequent few months. Further, according to $\mathrm{WHO}$, the dissemination of this virus has been observed with 111,102, 016 confirmed cases and 2462,911 confirmed deaths in 223 countries, areas or territories as on 22 February $2021 .^{1}$

Coronaviruses (CoVs) are a large family of viruses, several of which cause respiratory diseases in humans, from the common cold to more rare and serious diseases, such as the Severe Acute Respiratory Syndrome (SARS) and the Middle East respiratory syndrome (MERS), both of which have high mortality rates and were detected for the first time in 2003 and 2012, respectively. CoVs are divided into four genera: alpha-, beta-, gamma- and delta-CoV. All CoVs currently known cause disease in humans belonging to the alpha- or the beta-CoV. ${ }^{2}$ Symptoms of COVID-19 are variable, but often include fever, cough, fatigue, breathing difficulties, and loss of smell and taste. Symptoms begin one to fourteen days after exposure to the virus. Of those people who develop noticeable symptoms, most (81\%) develop mild to moderate symptoms (up to mild pneumonia), while $14 \%$ develop severe symptoms (dyspnea, hypoxia, or more than $50 \%$ lung involvement on imaging), and $5 \%$ suffer critical symptoms (respiratory failure, shock, or multi-organ dysfunction) [6]. At least a third of the people who are infected with the virus remain asymptomatic and do not develop noticeable symptoms at any point in time, but they still can spread the disease. ${ }^{3-5}$

SARS-CoV-2 is thought to have an animal (zoonotic) origin. Genetic analysis has revealed that the
Cite this article : Gupta BM, Dhawan SM, Ahmed KKM, Mamdapur GM. Global Research on COVID-19 Disease: A Scientific Assessment of Publications during 2020-21. Int J Med Public Health. 2021;11(2):76-84. 
coronavirus genetically clusters with the genus Betacoronavirus, in subgenus Sarbecovirus (lineage B) together with two bat-derived strains. The structural proteins of SARS-CoV-2 include membrane glycoprotein $(\mathrm{M})$, envelope protein $(\mathrm{E})$, nucleocapsid protein $(\mathrm{N})$, and the spike protein $(\mathrm{S}){ }^{6}$

Although COVID-19 mainly affects respiratory and immune systems, other systems likely to be hit are cardiovascular, urinary, gastrointestinal tract, reproductive systems and integumentary systems (skin, hair, nails, and exocrine glands). It is believed that virus can damage almost every part of the body including kidneys, the brain, small intestine and rectum. In respiratory system, COVID-19 can affect the upper respiratory tract (sinuses, nose, and throat) and the lower respiratory tract (windpipe and lungs). The lungs are the organs most affected by COVID-19 because the virus accesses host cells via the enzyme angiotensin-converting enzyme 2 (ACE2), which is most abundant in type II alveolar cells of the lungs. ${ }^{7}$

The effects and impact of COVID-19 has put unprecedented pressures on healthcare systems worldwide. Although the numbers of confirmed cases and deaths continue to rise, the scientific research community has promptly responded and a number of COVID-19-related clinical trials are currently underway. Research and scientific inquisitions are of paramount importance in the wake of pandemics such as COVID-19. The absence of clinically proven curative treatments or vaccines (now available) coupled with paucity in our understanding of the disease and long term clinical squeal fuels the need for further research to help us better combat the current pandemic.

As COVID-19 spreads rapidly, the research community has been active in publishing novel articles on this dreadful disease. Therefore, it is important to understand the global scientific output of COVID-19 right from the early stage of the outbreak till today. Thus, to track the current hotspots and highlight future directions, we performed a bibliometric analysis to obtain an approximate scenario of COVID-19 research to date. Such efforts will improve clinicians' understanding of the large and growing body of evidence on the topic.

Bibliometrics studies map various disciplines to identify their research trends, discover knowledge structures and monitor research performance at global, national, institutional and individual level. These studies also analyses global research on different metrics of quantity (output) and quality (performance) and by using relational indicators describe relationships between research networks, teams, institutions, and countries. In recent years, bibliometrics have been used to provide strong insights into several subject fields linked to COVID-19 research..$^{8-17}$ But most of these bibliometric studies on COVID-19 research are based on a limited data sets covering ( 2 to 6 months) publications period. Till date, not a single study has been undertaken that attempts to evaluate the status of COVID-19 research in a comprehensive way by covering publication data since the outbreak of COVID-19 disease till today. It is to fill this gap that a comprehensive study on this topic has been planned. This study will therefore attempt to examine and assess the global publications on COVID-19 research since the early stage of the COVID-19 outbreak till date (covering around 13 month publications data) using bibliometric methods. The study will attempt to identify the key countries, organizations and authors in global research, discover global collaborative trends in COVID-19 research, bring out leading source journals on this theme, identify highly cited publications and their bibliometric characteristics, and explore prominent research areas (broad and narrow subject areas) in COVID-19 research. The results from the study will provide policy-makers and scholars a valuable resource that they could apply to identify national research priorities and draw up future plans in COVID-19 research.

\section{MATERIALS AND METHODS}

The study sourced publications and citations data related to COVID-19 from Scopus database on 15 February 2021. The fact that the Scopus database is the largest abstract and citation database of peer-reviewed scientific literature; it was decided to use it as the bibliometric resource for sourcing data for the present study. A comprehensive search was performed using a set of keywords (shown below) tagged to "Title" or "Keyword" tags in the Scopus database, and limiting the search results to publication years 2020-2021. The search yielded a total of 1, 03, 054 hits. All the data records relevant to COVID-19 were downloaded from the Scopus database. Excel as a tool was used to statistically analyse the total publications output on a series of bibliographic variables.

TITLE (“COVID 19" OR "2019 novel coronavirus" OR "coronavirus 2019” OR “coronavirus disease 2019” OR “2019-novel CoV” OR "2019 related to COVID-19 AND SHOWN BELOWcov" OR covid 2019 OR covid19 OR “corona virus 2019” OR ncov-2019 OR ncov2019 OR "nCoV 2019" OR 2019-ncov OR covid-19 OR "Severe acute respiratory syndrome coronavirus 2" OR "SARS-CoV-2") OR KEY (“COVID 19" OR "2019 novel coronavirus" OR "coronavirus 2019” OR "coronavirus disease 2019” OR “2019-novel CoV” OR "2019 ncov” OR covid 2019 OR covid19 OR “corona virus 2019” OR ncov-2019 OR ncov2019 OR "nCoV 2019" OR 2019-ncov OR covid-19 OR "Severe acute respiratory syndrome coronavirus 2" OR "SARS-CoV-2") AND (LIMIT-TO (PUBYEAR, 2021) OR LIMIT-TO (PUBYEAR, 2020)).

\section{RESULTS}

\section{Overall}

As seen from Scopus database, a total of 103,054 publications $(2020=87290 ; 2021=15764)$ related to COVID-19 had appeared from the early stage of the COVID-19 outbreak till 15 February 2021. Of the global $(103,054)$ publications in the subject, a $32.11 \%$ share $(33087)$ resulted from research projects sponsored by $1000+$ national and international funding agencies. The top 20 funding agencies that supported research projects in this area are listed in Table 1.

In terms of distribution of global output by document type, it was seen that the bulk of the research output (55.18\%) on COVID-19 had appeared as articles, followed by letters and reviews (15.70\% and $11.31 \%)$, notes and editorials (7.63\% and $6.82 \%)$, short surveys and erratum $(1.51 \%$ and $1.06 \%$ ) and others accounted for a less than $1 \%$ share each: book chapters and data papers ( $0.59 \%$ each), books $(0.01 \%)$, etc. Most of the research output (a 94.13\% share) appeared in English language, followed by Spanish (2.35\%), Chinese, French and German (1.22\%, $1.11 \%$ and $1.04 \%)$, Portuguese, Russian, Italian $(0.54 \%, 0.43 \%$ and $0.35 \%)$, and rest in 31 other languages.

\section{Most Productive Countries in COVID-19 Research}

The USA published the largest number of publications on COVID-19 (25.56\% share), followed by China and U.K (10.77\% and $10.70 \%)$. The others in the list of top 20 most productive countries are Italy (9.07\%), India (6.95\%), Spain, Canada, Germany, France, Australia, Brazil and Iran (from $2.46 \%$ to $4.23 \%$ ), and Turkey, Switzerland, Netherlands, Japan, Saudi Arabia, Singapore, Belgium and South Korea (from 1.24\% to $1.97 \%$ ). The top 1-10 countries and top 11-20 countries together published 85,458 (82.93\%) and 18,729 (18.17\%) documents (Table 2).

In all, a total of 200 countries had participated in the COVID-19 research. But the distribution of their research output by country of publication is 
Table 1: Leading Funding Agencies Supporting Research on COVID-19.

$\begin{array}{ccccccc}\text { S.No } & \text { Funding Agencies } & \text { Pub. } & \text { S.No } & \text { Funding Agencies } & \text { Pub. } \\ \text { Count }\end{array}$

quite skewed. For instance, 106 countries published 1-50 papers each, 20 countries 51-100 papers each, 38 countries 101-494 papers each, 21 countries 1001-4357 papers each, 2 countries 7158-9350 papers each, 2 countries 11000-11104 papers each and 1 country 26339 papers.

\section{Research Collaboration among top 15 countries}

Figure 1 displays country collaborations among top 15 most productive countries. In the network diagram, each node represents a country. The node size and font size corresponds to publications output of the country. The Figure depicts countries in two clusters. Cluster 1 in apricot colour shows nine countries - USA, China, UK, Canada, India, Brazil, Iran, Australia, and turkey. Cluster 2 in brick colour shows six countries - Italy, Germany, France, Spain, Netherlands, and Switzerland. The thickness of the lines between countries indicates the degree of their collaboration in research. In cluster 1, the USA - U.K. lead in research collaboration with 1817 linkages, followed by USA - China (1519 links), USA - Canada (1292 links), USA - Australia (921 links), U.K.- Australia (775 links), USA - India (699 links), China - U.K (612 links), U.K.- Canada (608 links), USA - Iran (321 links), China - Canada (308 links). Inter-cluster collaboration instances include USA - Italy (1346 links), U.K. - Italy (1043 links), USA - Germany (801 links), USA - Switzerland (559 links), U.K. - Spain (550 links) U.K. - France (533 links), USA - Netherland (494 links), U.K.-Germany (682 links), USA-France (672 links), and USASpain (639 links). In cluster 2, Italy and Germany dominate in research collaboration with 596 links followed by Italy - France (554 links), Italy - Switzerland (413 links), and Italy - Spain (664 links).

\section{COVID-19 Research by Population Age Groups}

On classifying COVID-19 output by age groups, it was found that the maximum impact of COVID-19 was on "Adults" (15406 papers and $14.95 \%$ share), followed by "Middle Aged" (10910 papers and 10.59\%

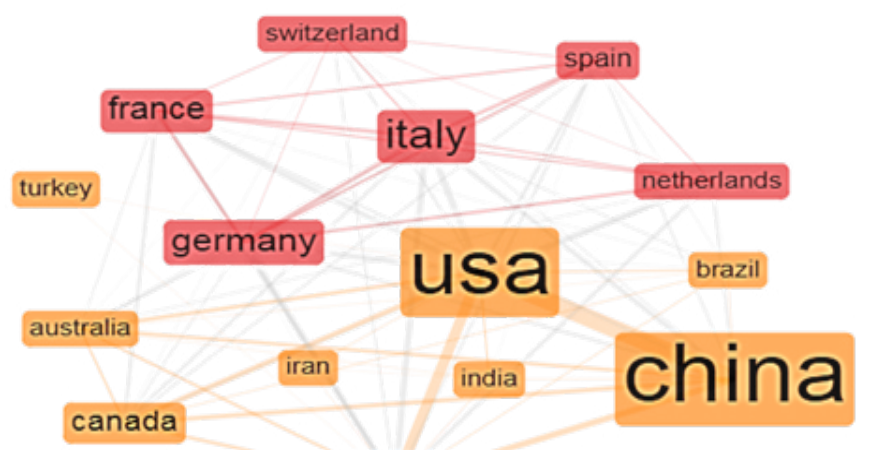

\section{united kingdom}

Figure 1: Country Collaboration Networks Chart.

share), "Aged" (10160 papers and 9.86\% share), "Adolescents" (3735 papers and $3.62 \%$ share) and "Children" (1596 papers and $1.55 \%$ share).

\section{Subject-Wise Distribution}

As per the subject classification of Scopus database, the total COVID-19 publication output is distributed across 21 broad subjects. Of these, Medicine accounted for the largest share (71.44\%), followed by Biochemistry, Genetics and Molecular Biology (10.61), Social Sciences (9.70\%), Immunology and Microbiology (6.71\%), Nursing (4.41\%), Pharmacology, Toxicology and Pharmaceutics (4.38\%), Environment Science $(4.02 \%)$ and other subjects contributing from $0.56 \%$ to $3.22 \%$ in global publication share respectively. The subject-wise distribution of COVID-19 reflects the multidisciplinary nature of research as well as its impact in different subjects (Table 3, Figure 2). 
Table 2: Geographical Distribution of Global Output on COVID-19.

\begin{tabular}{|c|c|c|c|c|c|c|c|}
\hline S.No & $\begin{array}{l}\text { Name } \\
\text { of the } \\
\text { Country }\end{array}$ & $\begin{array}{l}\text { No. of } \\
\text { Papers } \\
\text { (TP) }\end{array}$ & $\%$ TP & S.No & Name of the country & $\begin{array}{c}\text { No. of } \\
\text { Papers (TP) }\end{array}$ & $\%$ TP \\
\hline 1 & USA & 26339 & 25.56 & 13 & Turkey & 2034 & 1.97 \\
\hline 2 & China & 11104 & 10.77 & 14 & Switzerland & 1988 & 1.93 \\
\hline 3 & U.K. & 11029 & 10.70 & 15 & Netherlands & 1850 & 1.80 \\
\hline 4 & Italy & 9350 & 9.07 & 16 & Japan & 1660 & 1.61 \\
\hline 5 & India & 7158 & 6.95 & 17 & Saudi Arabia & 1524 & 1.48 \\
\hline 6 & Spain & 4357 & 4.23 & 18 & Singapore & 1381 & 1.34 \\
\hline 7 & Canada & 4321 & 4.19 & 19 & Belgium & 1287 & 1.25 \\
\hline 8 & Germany & 4041 & 3.92 & 20 & South Korea & 1273 & 1.24 \\
\hline 9 & France & 3971 & 3.85 & & Total of top 10 countries & 85458 & 82.93 \\
\hline 10 & Australia & 3788 & 3.68 & & Total of top 11-20 countries & 18729 & 18.17 \\
\hline 11 & Brazil & 3201 & 3.11 & & $\begin{array}{c}\text { Global total of top } 20 \\
\text { countries }\end{array}$ & 104187 & 100.66 \\
\hline 12 & Iran & 2531 & 2.46 & & Global total & 103504 & \\
\hline
\end{tabular}

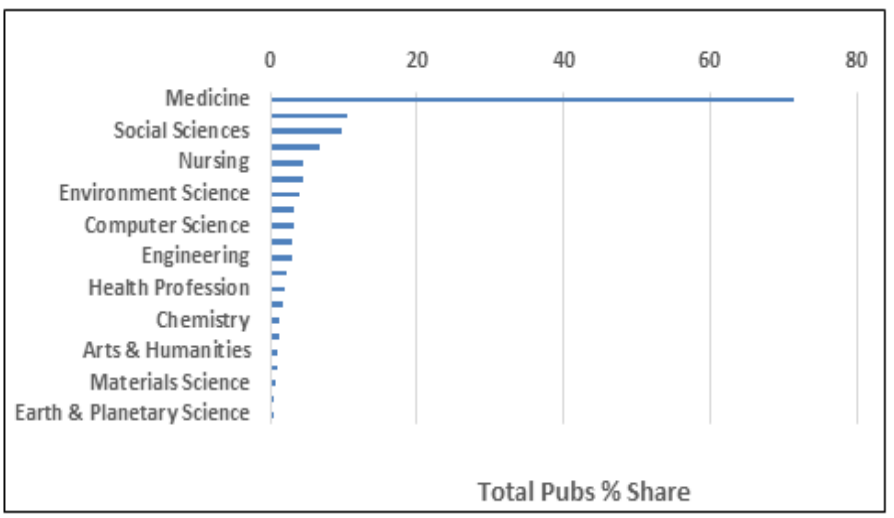

Figure 2: Distribution of COVID-19 Research Output by Subject Areas 2020-21.

\section{Distribution of COVID-19 Output by Broad Sub-fields}

Using different key-word strategies, the COVID-19 research output was studied under various sub-fields and by nature of research. Treatment methods (17.94\%) account for the largest publication share (11.90\%) of the COVID-19 publication output, followed by clinical studies (11.90\%), complications (7.06\%), epidemiological studies $(3.60 \%)$ and pathophysiology (3.55\%), etc.

Under clinical studies, Virological studies account for the largest publication share $(9.58 \%)$, followed by respiratory studies $(6.97 \%)$, molecular studies $(3.11 \%)$, genetics studies $(3.09 \%)$, biological studies (2.85\%), immunological studies (1.30\%), etc. Under treatment studies, drug therapy accounts for the largest publication share (9.90\%), followed by vaccine $(1.77 \%)$, oxygen therapy $(1.19 \%)$, immunosuppressive therapy $(0.81)$ and convalescent plasma $(0.46 \%)$, etc. The maximum impact of COVID-19 was observed on environment studies $(4.02 \%)$, followed by mental health $(3.22 \%)$, neurosciences $(2.99 \%)$, economy (1.76\%), cardiovascular disease (1.74\%) and dentistry (1.01\%) (Table 4).

\section{COVID-19 Output by Significant Keywords}

As seen from the Scopus database, 185 significant keywords seem to identify and define global literature on COVID-19. The co-occurrence of these keywords in more than one document provide a secondary approach to identify broad research trends in the investigation and
Table 3: Covid-19 Publications Distribution by Broad Subjects.

$\begin{array}{cccc}\text { S.No } & \text { Broad Subject } & \text { TP } \\ 1 & \text { Medicine } & 73625 & 71.44 \\ 2 & \text { Biochemistry, Genetics and Molecular Biology } & 10938 & 10.61 \\ 3 & \text { Social Sciences } & 9999 & 9.70 \\ 4 & \text { Immunology and Microbiology } & 6912 & 6.71 \\ 5 & \text { Nursing } & 4549 & 4.41 \\ 6 & \text { Pharmacology, Toxicology and Pharmaceutics } & 4512 & 4.38 \\ 7 & \text { Environment Science } & 4143 & 4.02 \\ 8 & \text { Psychology } & 3323 & 3.22 \\ 9 & \text { Computer Science } & 3262 & 3.17 \\ 10 & \text { Neuroscience } & 3084 & 2.99 \\ 11 & \text { Engineering } & 3043 & 2.95 \\ 12 & \text { Agricultural and Biological Sciences } & 2408 & 2.34 \\ 13 & \text { Health Profession } & 2091 & 2.03 \\ 14 & \text { Economics, Econometrics and Finance } & 1813 & 1.76 \\ 15 & \text { Chemistry } & 1297 & 1.26 \\ 16 & \text { Physics and Astronomy } & 1260 & 1.22 \\ 17 & \text { Arts and Humanities } & 906 & 0.88 \\ 18 & \text { Energy } & 906 & 0.88 \\ 19 & \text { Materials Science } & 827 & 0.80 \\ 20 & \text { Veterinary Science } & 605 & 0.59 \\ 21 & \text { Earth and Planetary Science } & 581 & 0.56 \\ & \text { Global Total } & 103054 & 100.00\end{array}$

treatment of the COVID-19, and to assess its impact on different subject fields and on various human organs. These keywords have been classified under broad subject headings along with the frequency of keyword occurrence (Table 5).

\section{Productivity of Most Productive Organizations}

The top 50 organizations involved in COVID-19 research together contributed 32, 653 papers (31.69\% share) and 674442 citations, with 
Table 4: Sub-field Distribution of Papers on COVID-19 Research.

\begin{tabular}{|c|c|c|c|}
\hline S.No & Subject Areas & Pub. Count & \% Share \\
\hline \multirow[t]{8}{*}{1} & Clinical Studies & 12267 & 11.90 \\
\hline & Molecular Aspects & 3202 & 3.11 \\
\hline & Immunological Aspects & 1342 & 1.30 \\
\hline & Virological Aspects & 9874 & 9.58 \\
\hline & Biological Aspects & 2940 & 2.85 \\
\hline & Respiratory Aspects & 7183 & 6.97 \\
\hline & Diagnostic Aspects & 2543 & 2.47 \\
\hline & Genetic Aspects & 3182 & 3.09 \\
\hline 2 & Complication & 7279 & 7.06 \\
\hline 3 & Epidemiology & 3708 & 3.60 \\
\hline 4 & Pathophysiology & 3661 & 3.55 \\
\hline \multirow[t]{9}{*}{5} & Treatment & 18489 & 17.94 \\
\hline & Convalescent Plasma & 470 & 0.46 \\
\hline & Treatment Outcome & 2732 & 2.65 \\
\hline & Vaccine & 1824 & 1.77 \\
\hline & Drugs Therapy & 10200 & 9.90 \\
\hline & Oxygen Therapy & 1231 & 1.19 \\
\hline & Immunosupportive Therapy & 839 & 0.81 \\
\hline & Biological Therapy & 1193 & 1.16 \\
\hline & Anti-monoclonal Therapy & 860 & \\
\hline \multirow[t]{9}{*}{6} & Impact of COVID-19 on & & \\
\hline & Diabetes & 2516 & 2.44 \\
\hline & Cardiovascular Disease & 1788 & 1.74 \\
\hline & Mental Health & 3322 & 3.22 \\
\hline & Neurosciences & 3084 & 2.99 \\
\hline & Dentistry & 1043 & 1.01 \\
\hline & Economy & 1813 & 1.76 \\
\hline & Environment & 4143 & 4.02 \\
\hline & & 103054 & 100.00 \\
\hline
\end{tabular}

an average of 20.65 citations per paper since their publication. Their publications productivity per organization varied from 425 to 1755 papers. Of the top 50 organizations, 18 were from USA, 7 from Australia, 6 each from China, Italy and U.K., 4 from France, 2 from Canada and 1 each from Brazil, India, Iran, Australia, Singapore, Iran and Hong Kong. Seventeen of the top 50 organizations contributed publications above the group-50 average of 653.06 papers per organization. Only nine of these 50 organizations registered their citation performance above group- 50 average of 20.65 citations per organization. The top 10 organizations each are listed in terms of publications productivity and citation impact per paper in Table 6.

\section{Research Collaboration among top 15 organizations}

Figure 3 displays collaborations among top 15 organizations in four clusters. Cluster 1 in chocolate color shows four organizations - Harvard Medical School, USA, Brigham Women's Hospital, USA, University of Toronto, Canada, Icahn School of Medicine at Mount Sinai, USA. Clusters 2 in light blue colour shows three organizations - Cluster 3 in lime colour shows two organizations, and cluster 4 in blue colour shows four organizations. The box size and font size of organizations in each cluster is proportional to their collaborative publications. Huazhong

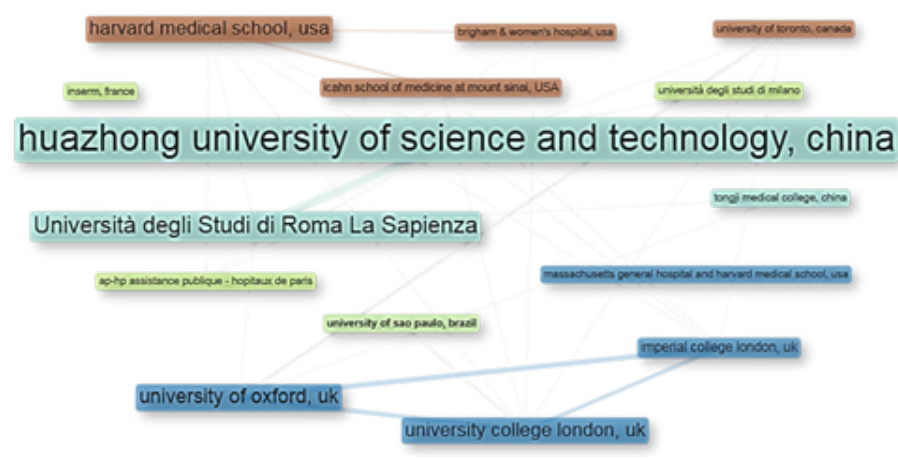

Figure 3: Organization Collaboration Network Chart.

University of S\&T, China - Tongji Medical College, China registered the highest number of collaborative linkages (1222), followed Harvard Medical School, USA - Brigham and Women's Hospital, USA (570 links), Harvard Medical School, USA - Massachusetts General Hospital, USA (557 links), INSERM, France - AP-HP Assistance Publique - Hopitaux de Paris (464 links), Massachusetts General Hospital, USA - Brigham and Women's Hospital, USA (163 links), University of Oxford, U.K. Imperial College, London, U.K. (100 links), Harvard Medical School, USA - Icahn School of Medicine at Mount Sinai, USA (63 links), etc.

\section{Productivity of Most Productive Authors}

The top 50 authors involved in research in COVID-19 together contributed 2635 papers (2.56\% share) and 84889 citations, with an average of 32.22 citations per paper since their publication. Their publications productivity per author varied from 37 to 152 papers. Of the top 50 authors, 10 were from U.K., 8 from Italy, 7 from USA, 5 from India, 3 each from France and Hong Kong, 2 each from Belgium and China and 1 each from Columbia, Iran, Malaysia, Switzerland, Canada, Nepal, Saudi Arabia, Indonesia and Singapore. Thirteen of top 50 authors contributed publications above group-50 average ( 52.7 publications per author). Fourteen of top 50 authors registered their citation performance above the group-50 average (32.22 citations per author). The top 10 authors each in terms of publication productivity and citation impact per papers is listed in Table 7 .

\section{Most Productive Journals}

The top 50 journals involved in research in COVID-19 together contributed 16482 papers and 321093 citations, with average citations per paper of 19.48 . These 50 journals contributed 179 to 261 papers each, together they account for $16.27 \%$ share of global output. Sixteen of top 50 journals contributed papers above the group-50 average (329.64 papers per journal). Thirteen of top 50 journals registered their citation performance above the group-50 average ( 19.48 per journal). The top 10 journals each in terms of publication productivity and citation impact per papers are listed in Table 8 .

\section{Highly Cited Papers}

A total of 1360 papers (1.32\% share of 103054 global outputs) received 100 to 11778 citations per paper since their publication. These 1360 paper (assumed as highly-cited papers) together received 175386 citations, averaging to 128.96 citations per paper. Of the 1360 high cited papers, 758 were in citations range 100-200, 431 in citation range of 201-500, 108 in citation range 501-999, 57 in citation range of 1001-4999, 5 papers in citation range 5000-7794 and 1 paper 11778 citations. A list of top 10 high cited papers is given in Table 9. 


\section{Table 5: Distribution of Significant Keywords under Broad Sub-fields Clusters.}

\begin{tabular}{|c|c|c|}
\hline S.No & Subject & Keywords (along with the frequency of their occurrence \\
\hline 1 & COVID-19 & Coronavirus Disease 2019 (38605), Coronavirus Infection (36847), Pandemic (35944), COVID-19 (34726), SARS-CoV-2(32066) \\
\hline 2 & Epidemiology & Epidemiology (6409), Prevalence (3362), Incidence (2565) \\
\hline 4 & Pathophysiology & Pathophysiology (3346), Physiology (2407) \\
\hline 5 & $\begin{array}{l}\text { Respiratory } \\
\text { Aspects }\end{array}$ & $\begin{array}{c}\text { Virus Pneumonia (368243), Adult Respiratory Distress Syndrome (3009), Dyspnea (3395S), Severe Acute Respiratory Syndrome } \\
\text { (2293), Respiratory Failure (1722), Lung (1625), Lung Embolism (891), Chronic Obstructive Lung Disease (805), Respiratory } \\
\text { Disease (460), Asthma (306), Lung Embolism (243) Pleura Effusion (191), Lung Fibrosis (102), Lung Parenchyma (101), Pulmonary } \\
\text { Hypertension (90) }\end{array}$ \\
\hline 6 & Molecular Aspects & Molecular Docking (666), Molecular Dynamics (320), Molecular Models (255), Molecular Evolution (144) \\
\hline 7 & Biological Aspects & Biological Markers (1025), Biological Models (426), Computational Biology (205) \\
\hline 8 & $\begin{array}{l}\text { Immunological } \\
\text { Aspects }\end{array}$ & $\begin{array}{c}\text { Immunology (4312), Cytokine Storm (2732), Immune Response (1878), Immunoglobulin G (1260), Immunoglobulin (999), } \\
\text { Immunoglobulin M (817), Immunotherapy (620), Immunomodulation (388), Immunosuppressive Treatment (141) Monoclonal } \\
\text { Antibodies (857) }\end{array}$ \\
\hline 11 & Gastro & $\begin{array}{c}\text { Gastrointestinal Symptoms (493), Gastrointestinal Disease (312), Liver Injury (339), Liver Disease (330), Liver Transplantation (230), } \\
\text { Jaundice (40) }\end{array}$ \\
\hline 12 & Mental Health & $\begin{array}{c}\text { Psychology (4553), Mental Health (3322), Depression (2212), Anxiety (1879), Mental Stress (1174), Stress (929), Mental Disease } \\
\text { (604), Anxiety Disorder (349) Distress Syndrome(470) Psychological Adaptation (270), Sleep Disorders (297), Sleep Wake Disorders } \\
\text { (77), Insomnia (411) }\end{array}$ \\
\hline 13 & Brain & Brain Hemorrhage (327), Brain Ischemia (356) \\
\hline 14 & Heart & Cardiovascular Disease (1788), Heart Muscle Injury (378), Heart Failure (243) Myocarditis (543), Ischemic Heart Disease (268) \\
\hline 15 & $\begin{array}{l}\text { Reproductive } \\
\text { Organs }\end{array}$ & $\begin{array}{l}\text { Pregnancy (1687), Pregnancy Complications (798), Pregnancy Outcome (310), Reproductive Health (148), Prostate Cancer (216), } \\
\text { Infertility therapy (62), Ovary Cancer (56), Infertility (46), Testis (36), obstetric Delivery (208) }\end{array}$ \\
\hline 19 & Pancreases & Diabetes Mellitus (2516), Acute Pancreatitis (72), Pancreas Cancer (62), Pancreatitis (36), Pancreas Disease (19) \\
\hline 20 & Drug Therapy & $\begin{array}{l}\text { Drug Therapy (1070) Hydroxychloroquine (4253), Azithromycin (2086), Remdesivir (2066), Lopinavir Plus Ritonavir (1954), } \\
\text { Tocilizumab (1864), Chloroquine (1638), Drug Repositioning (792), Anti-viral Therapy (740). Favipiravir (704) }\end{array}$ \\
\hline 21 & Rectum & Rectum Hemorrhage (43), Retum Cancer (37) \\
\hline 22 & Plasma Therapy & Convalescent plasma (605), Passive Immunization (389) \\
\hline 23 & Vaccine & Vaccination (1095), Virus Vaccine (1089), COVID-19 Vaccine (10692), Vaccine (700) \\
\hline 24 & $\begin{array}{l}\text { Monoclonal } \\
\text { Antibody Therapy }\end{array}$ & Monoclonal Antibody (860), Humanized Monoclonal Antibody (449) \\
\hline 25 & Blood Clotting & Blood clotting Disorders (117), Venus Thromboembolism (277)., Intravascular Clotting (179), Thrombosis (635) \\
\hline 26 & Urinary & Urinary Tract Infection(165), Urology Diseases (65), Urological Surgical (41) \\
\hline 27 & $\begin{array}{l}\text { Preventive } \\
\text { Measures }\end{array}$ & $\begin{array}{l}\text { Quarantine (4668), Social Distancing (2567), Personal Protective Equipment (1947), Social Isolation (1888), Face Masks (496), Hand } \\
\text { Wash (1512), Disinfect Surfaces }\end{array}$ \\
\hline 28 & Environment & $\begin{array}{l}\text { Air Pollution (315), Atmosphere Pollution (208), Air Pollutants (174), Sustainability (152), Environmental Monitoring (139), } \\
\text { Environmental Impact (117), Climate Change (108) }\end{array}$ \\
\hline 29 & Dental Science & $\begin{array}{l}\text { Dentistry (123), Dental Procedure (79), Dental Care (64), Dental Practice (32) Maxillofacial Surgery(36), Saliva (34), Oral Health } \\
\text { (32) }\end{array}$ \\
\hline 30 & Machine Learning & $\begin{array}{l}\text { Deep Learning (308), Machine Learning (210), Artificial Intelligence (185), Learning Systems (172), Convolution Neural Networks } \\
\text { (59), Deep Neural Networks (54), Decision Trees (49), Neural Networks (47), Support Vector Machines (46), Decision Support } \\
\text { System (36) }\end{array}$ \\
\hline
\end{tabular}


Table 6: Profile of Top 10 Most Productive and 10 Most Cited Organizations.

\begin{tabular}{|c|c|c|c|c|}
\hline S.No & Name of Organization & TP & TC & CPP \\
\hline \multicolumn{5}{|c|}{ Top 10 Most Productive Organizations } \\
\hline 1 & Harvard Medical School, USA & 1755 & 25533 & 14.55 \\
\hline 2 & $\begin{array}{c}\text { Huazhong University of S\&T, } \\
\text { China }\end{array}$ & 1344 & 70983 & 52.81 \\
\hline 3 & Tongji Medical College, China & 1267 & 69301 & 54.70 \\
\hline 4 & INSERM, France & 1206 & 19065 & 15.81 \\
\hline 5 & University of Toronto, Canada & 1138 & 12206 & 10.73 \\
\hline 6 & University of Milan, Italy & 932 & 14567 & 15.63 \\
\hline 7 & University of Oxford, U.K. & 903 & 18884 & 20.91 \\
\hline 8 & University College, London, U.K. & 877 & 17950 & 20.47 \\
\hline 9 & $\begin{array}{c}\text { Sapienza University of Rome, } \\
\text { Italy }\end{array}$ & 877 & 8040 & 9.17 \\
\hline 10 & $\begin{array}{c}\text { Massachusetts General Hospital, } \\
\text { USA }\end{array}$ & 848 & 10130 & 11.95 \\
\hline \multicolumn{5}{|c|}{ Top 10 Most Cited Organizations } \\
\hline 1 & $\begin{array}{l}\text { Chinese Academy of Medical } \\
\text { Sciences and Peking Union } \\
\text { Medical College, China }\end{array}$ & 438 & 42666 & 97.41 \\
\hline 2 & University of Kong Hong & 479 & 39359 & 82.17 \\
\hline 3 & $\begin{array}{c}\text { Renmin Hospital of Wuhan } \\
\text { University, China }\end{array}$ & 439 & 26287 & 59.88 \\
\hline 4 & Tongji Medical College, China & 1267 & 69301 & 54.70 \\
\hline 5 & $\begin{array}{l}\text { Huazhong University of S\&T, } \\
\text { China }\end{array}$ & 1344 & 70983 & 52.81 \\
\hline 6 & Fudan University & 431 & 16498 & 38.28 \\
\hline 7 & Ministry of Education, China & 460 & 12668 & 27.54 \\
\hline 8 & $\begin{array}{l}\text { University of Washington, } \\
\text { Seattle, USA }\end{array}$ & 631 & 15593 & 24.71 \\
\hline 9 & University of Oxford, U.K. & 903 & 18884 & 20.91 \\
\hline 10 & University of Cambridge, U.K. & 439 & 8991 & 20.48 \\
\hline
\end{tabular}

${ }^{*} \mathrm{TP}=$ Total publications; $\mathrm{TC}=$ Total citations; $\mathrm{CPP}=$ Citations per paper

Among the various participating countries in COVID-19 research, China contributed the largest number (535) of highly-cited papers, followed by United States (449 papers), U.K. (!92 papers), Italy (162 papers), Germany (91 papers), France (83 papers), Canada (67 papers), Hong Kong (55 papers), Netherland (51 papers), Switzerland (50 papers), Singapore (49 papers), Spain and Australia (48 papers each), India (36 papers), Brazil and South Korea (29 papers each), Japan (25 papers), Sweden (22 papers), Belgium (21 papers), Iran (19 papers), Saudi Arabia (18 papers), Austria (15 papers), South Africa (11 papers), Russia Federation and Ireland (10 papers each), Poland and Mexico ( 9 papers each), Turkey (7 papers), Pakistan (6 papers), etc.

The top organizations in terms of publication productivity of highlycited papers were : Huazhong University of S\&T, China (101 papers), Tongji Medical College, China (97 papers), University of Kong Hong (53 papers), Harvard Medical School, USA (51 papers), Chinese Academy of Medical Sciences and Peking Union Medical College, China (42 papers), Fudan University, China (41 papers), INSERM, France and Renmin Hospital of Wuhan University, China (39 papers each), Imperial College, London, U.K. (36 papers), University of Oxford, U.K. (34 papers), University College, London, U.K. (30 papers), Brigham and Women's
Table 7: Profile of Top 10 Most Productive and 10 Most Cited Authors.

\begin{tabular}{|c|c|c|c|c|c|}
\hline S.No & $\begin{array}{c}\text { Name of the } \\
\text { Author }\end{array}$ & $\begin{array}{l}\text { Affiliation of the } \\
\text { Author }\end{array}$ & TP & TC & CPP \\
\hline \multicolumn{6}{|c|}{ Top 10 Most Productive Authors } \\
\hline 1 & V. Wiwanitlit & $\begin{array}{l}\text { Dr D.Y Patil University, } \\
\text { Pune }\end{array}$ & 152 & 541 & 3.56 \\
\hline 2 & E. Mahase & $\begin{array}{l}\text { British Medical Journal, } \\
\text { U.K. }\end{array}$ & 135 & 938 & 6.95 \\
\hline 3 & G. Iacobucci & $\begin{array}{l}\text { British Medical Journal, } \\
\text { U.K. }\end{array}$ & 108 & 299 & 2.77 \\
\hline 4 & G. Lippi & $\begin{array}{l}\text { University of Verona, } \\
\text { Italy }\end{array}$ & 103 & 3965 & 38.50 \\
\hline 5 & K. Dhama & $\begin{array}{c}\text { Indian Veterinary } \\
\text { Research Institute, } \\
\text { Bareilly, India }\end{array}$ & 83 & 1608 & 19.37 \\
\hline 6 & A. Rimmer & $\begin{array}{l}\text { British Medical Journal, } \\
\text { U.K. }\end{array}$ & 70 & 128 & 1.83 \\
\hline 7 & $\begin{array}{l}\text { A.J.Rodruquez- } \\
\text { Morales. }\end{array}$ & $\begin{array}{c}\text { Technological } \\
\text { University of Pereira, } \\
\text { Columbia }\end{array}$ & 68 & 1738 & 25.56 \\
\hline 8 & N.Rezaei & $\begin{array}{l}\text { Tehran University of } \\
\text { Medical Sciences, Iran }\end{array}$ & 65 & 639 & 9.83 \\
\hline 9 & S.S.Hasan & $\begin{array}{c}\text { University of } \\
\text { Huddersfield, U.K. }\end{array}$ & 60 & 92 & 1.53 \\
\hline 10 & B.M.Henry & $\begin{array}{c}\text { Cincinnati Children's } \\
\text { Hospital Medical } \\
\text { Centre, USA }\end{array}$ & 58 & 1922 & 3.14 \\
\hline
\end{tabular}

Top 10 Most Cited Authors

\begin{tabular}{|c|c|c|c|c|c|}
\hline 1 & $\mathrm{Hu}, \mathrm{Y}$. & $\begin{array}{c}\text { Tongji Medical College, } \\
\text { China }\end{array}$ & 38 & 12153 & 319.82 \\
\hline 2 & L.Liu & $\begin{array}{l}\text { Second Affiliated } \\
\text { Hospital of Southern } \\
\text { University of S\&T, } \\
\text { China }\end{array}$ & 45 & 13676 & 303.91 \\
\hline 3 & J.F.W.Chan & $\begin{array}{l}\text { University of Hong } \\
\text { Kong }\end{array}$ & 40 & 6782 & 169.55 \\
\hline 4 & K.K.W.To & $\begin{array}{l}\text { University of Hong } \\
\text { Kong }\end{array}$ & 42 & 6946 & 165.38 \\
\hline 5 & J.C.Lagier & $\begin{array}{c}\text { The Research Institute } \\
\text { for Development (IRD), } \\
\text { France }\end{array}$ & 39 & 3141 & 80.54 \\
\hline 6 & P.Gautret & $\begin{array}{l}\text { The Research Institute } \\
\text { for Development (IRD), } \\
\text { France }\end{array}$ & 41 & 2717 & 66.27 \\
\hline 7 & Raoult, D. & $\begin{array}{c}\text { The Research Institute } \\
\text { for Development (IRD), } \\
\text { France }\end{array}$ & 39 & 2380 & 61.03 \\
\hline 8 & Leo, Y.S. & $\begin{array}{c}\text { Tan Tock Seng Hospital, } \\
\text { Singapore }\end{array}$ & 37 & 1883 & 50.89 \\
\hline 9 & A.Zangrillo & $\begin{array}{c}\text { IRCCS San Raffaele } \\
\text { Scientific Institute, Italy }\end{array}$ & 48 & 2393 & 49.85 \\
\hline 10 & Plebani, M. & $\begin{array}{l}\text { Azienda Ospedaliera di } \\
\text { Padova“(AOP), Italy }\end{array}$ & 38 & 1781 & 46.87 \\
\hline
\end{tabular}

${ }^{*} \mathrm{TP}=$ Total publications; $\mathrm{TC}=$ Total citations; $\mathrm{CPP}=$ Citations per paper 
Table 8: Profile of Top 10 Most Productive and Cited Journals.

\begin{tabular}{|c|c|c|c|c|}
\hline S.No & Name of the Journal & TP & TC & CPP \\
\hline \multicolumn{5}{|c|}{ Top 10 Most Productive Journals } \\
\hline 1 & $\begin{array}{l}\text { International Journal of } \\
\text { Environmental Research } \\
\text { and Public Health }\end{array}$ & 961 & 6119 & 6.37 \\
\hline 2 & $\mathrm{BMJ}$ & 854 & 9871 & 11.56 \\
\hline 3 & PLOS One & 781 & 2629 & 3.37 \\
\hline 4 & $\begin{array}{l}\text { Journal of Medical } \\
\text { Virology }\end{array}$ & 779 & 17070 & 21.91 \\
\hline 5 & BMJ Clinical Ed & 684 & 3106 & 4.54 \\
\hline 6 & The Lancet & 564 & 59956 & 106.30 \\
\hline 7 & $\begin{array}{l}\text { Journal of Infectious } \\
\text { Diseases }\end{array}$ & 530 & 8301 & 15.66 \\
\hline 8 & $\begin{array}{l}\text { JAMA-Journal of the } \\
\text { American Medical } \\
\text { Association }\end{array}$ & 482 & 36234 & 75.17 \\
\hline 9 & $\begin{array}{c}\text { New England Journal of } \\
\text { Medicine }\end{array}$ & 441 & 54145 & 122.78 \\
\hline 10 & Science & 427 & 13873 & 32.49 \\
\hline \multicolumn{5}{|c|}{ Top 10 Most Cited Journals } \\
\hline 1 & $\begin{array}{c}\text { New England Journal of } \\
\text { Medicine }\end{array}$ & 441 & 54145 & 122.78 \\
\hline 2 & The Lancet & 564 & 59956 & 106.30 \\
\hline 3 & $\begin{array}{l}\text { JAMA-Journal of the } \\
\text { American Medical } \\
\text { Association }\end{array}$ & 482 & 36234 & 75.17 \\
\hline 4 & $\begin{array}{c}\text { Journal of Medical } \\
\text { Virology }\end{array}$ & 779 & 17070 & 21.91 \\
\hline 5 & Science & 427 & 13873 & 32.49 \\
\hline 6 & Nature & 297 & 16476 & 55.47 \\
\hline 7 & BMJ & 854 & 9871 & 11.56 \\
\hline 8 & Lancet Infectious Diseases & 261 & 12566 & 48.15 \\
\hline 9 & $\begin{array}{l}\text { Science of the Total } \\
\text { Environment }\end{array}$ & 368 & 8074 & 21.94 \\
\hline 10 & Journal of Infection & 380 & 7724 & 20.33 \\
\hline
\end{tabular}

Hospital, USA (29 papers), Ministry of Education, China (28 papers), University of Milan and National University of Singapore (26 papers each).

Among authors participating in highly-cited papers, L. Liu of Second Affiliated Hospital of Southern University of S\&T, China) contributed the largest number of papers (19 papers), followed by Y. Liu (Second Affiliated Hospital of Southern Univ of S\&T, China) (10 papers), J.F.W. Chan (University of Hong Kong (10 papers), K.K.W. To (University of Hong Kong (10 papers), G. Lippi (University of Verona, Italy) (10 papers), Z. Zhang (Second Affiliated Hospital of Southern Univ of S\&T, China) (9 papers), Y. Ling (Shanghai Public Health Clinical Centre, China (9 papers), Y.Hu (Tongji Medical College, China) (7 papers), Y. Xiong (Zhongnan Hospital of Wuhan University, China) (7 papers), P.Du (Peking Union Medical College Hospital,(China (7 papers), K.Y.Yuen (University of Hong Kong) (7 papers), J. Zhao (Tongji Medical College, China) (7 papers), J.C. Lagier (The Research Institute for Development, France) (6 papers) and Y.S. Leo,(Tan Tock Seng Hospital, Singapore) (6 papers).
Table 9: List of Top 10 Highly Cited Papers.

\begin{tabular}{|c|c|c|c|c|}
\hline S.No & Authors & Title & Source & $\begin{array}{l}\text { Number } \\
\text { of } \\
\text { Citations }\end{array}$ \\
\hline 1 & $\begin{array}{l}\text { Huang, } \\
\text { C.A, et al. }\end{array}$ & $\begin{array}{c}\text { Clinical features of } \\
\text { patients infected with } \\
2019 \text { novel coronavirus } \\
\text { in Wuhan, China }\end{array}$ & $\begin{array}{l}\text { The Lancet, } 2020 \\
395 \text { (10223), pp. } \\
\text { 497-506 }\end{array}$ & 11832 \\
\hline 2 & $\begin{array}{l}\text { Guan, W. } \\
\text { et al. }\end{array}$ & $\begin{array}{l}\text { Clinical characteristics } \\
\text { of coronavirus disease } \\
2019 \text { in China, }\end{array}$ & $\begin{array}{l}\text { New England } \\
\text { Journal of } \\
\text { Medicine, 2020, } \\
382 \text { (18), pp. } \\
1708-1720\end{array}$ & 7838 \\
\hline 3 & $\begin{array}{l}\text { Wang, D. } \\
\text { et al. }\end{array}$ & $\begin{array}{l}\text { Clinical Characteristics } \\
\text { of } 138 \text { Hospitalized } \\
\text { Patients with } 2019 \\
\text { Novel Coronavirus- } \\
\text { Infected Pneumonia in } \\
\text { Wuhan, China }\end{array}$ & $\begin{array}{l}\text { JAMA - Journal } \\
\text { of the American } \\
\text { Medical } \\
\text { Association, } \\
\text { 2020, 323 (11), } \\
\text { pp. 1061-1069. }\end{array}$ & 6753 \\
\hline 4 & $\begin{array}{l}\text { Zhou, F. } \\
\text { et al. }\end{array}$ & $\begin{array}{l}\text { Clinical course and risk } \\
\text { factors for mortality of } \\
\text { adult inpatients with } \\
\text { COVID-19 in Wuhan, } \\
\text { China: a retrospective } \\
\text { cohort study. }\end{array}$ & $\begin{array}{l}\text { The Lancet, 2020, } \\
395 \text { (10229), pp. } \\
\text { 1054-1062. }\end{array}$ & 6547 \\
\hline 5 & $\begin{array}{l}\text { Zhu, N } \\
\text { et al. }\end{array}$ & $\begin{array}{l}\text { A novel coronavirus } \\
\text { from patients with } \\
\text { pneumonia in China, } \\
2019 .\end{array}$ & $\begin{array}{l}\text { New England } \\
\text { Journal of } \\
\text { Medicine, 2020, } \\
382 \text { (8), pp. } 727- \\
733 .\end{array}$ & 6449 \\
\hline 6 & $\begin{array}{l}\text { Chen, } \mathrm{N} \\
\text { et al. }\end{array}$ & $\begin{array}{l}\text { Epidemiological and } \\
\text { clinical characteristics } \\
\text { of } 99 \text { cases of } 2019 \text { novel } \\
\text { coronavirus pneumonia } \\
\text { in Wuhan, China: A } \\
\text { descriptive study. }\end{array}$ & $\begin{array}{l}\text { The Lancet, 2020, } \\
395 \text { (10223), pp. } \\
\text { 507-513. }\end{array}$ & 5851 \\
\hline 7 & $\begin{array}{l}\text { Zhou, P. } \\
\text { et al. }\end{array}$ & $\begin{array}{c}\text { A pneumonia outbreak } \\
\text { associated with a new } \\
\text { coronavirus of probable } \\
\text { bat origin }\end{array}$ & $\begin{array}{l}\text { Nature, } 2020, \\
579 \text { (7798), pp. } \\
270-273 .\end{array}$ & 4868 \\
\hline 8 & $\begin{array}{l}\text { Wu, } \mathrm{Z} \text { and } \\
\text { McGoogan, } \\
\text { J.M. }\end{array}$ & $\begin{array}{l}\text { Characteristics of and } \\
\text { Important Lessons from } \\
\text { the Coronavirus Disease } \\
2019 \text { (COVID-19) } \\
\text { Outbreak in China: } \\
\text { Summary of a Report } \\
\text { of } 72314 \text { Cases from } \\
\text { the Chinese Center for } \\
\text { Disease Control and } \\
\text { Prevention. }\end{array}$ & $\begin{array}{l}\text { JAMA - Journal } \\
\text { of the American } \\
\text { Medical } \\
\text { Association, } \\
2020,323 \text { (13), } \\
\text { pp. 1239-1242 }\end{array}$ & 4598 \\
\hline 9 & Li, Q. et al. & $\begin{array}{l}\text { Early transmission } \\
\text { dynamics in Wuhan, } \\
\text { China, of novel } \\
\text { coronavirus-infected } \\
\text { pneumonia. }\end{array}$ & $\begin{array}{l}\text { New England } \\
\text { Journal of } \\
\text { Medicine, 2020, } \\
382 \text { (13), pp. } \\
1199-1207\end{array}$ & 4332 \\
\hline 10 & $\begin{array}{c}\text { Hoffmann, } \\
\text { M. et al. }\end{array}$ & $\begin{array}{l}\text { SARS-CoV-2 Cell Entry } \\
\text { Depends on ACE2 } \\
\text { and TMPRSS2 and Is } \\
\text { Blocked by a Clinically } \\
\text { Proven Protease } \\
\text { Inhibitor. }\end{array}$ & $\begin{array}{c}\text { Cell, } 2020,181 \\
\text { (2), pp. } 271-280 .\end{array}$ & 3642 \\
\hline
\end{tabular}


Among the various journals contributing to highly-cited papers, New England Journal of Medicine contributed the largest number of papers (71), followed by The Lancet (63 papers), JAMA-Journal of the American Medical Association (55 papers), Journal of Medical Virology (41 papers), Science (31 papers), Nature (25 papers), BMJ (24 papers), Lancet Infectious Diseases (22 papers), Science of the Total Environment (21 papers), Journal of Infection (20 papers), Journal of Infectious Diseases (16 papers), Lancet Respiratory Medicine (15 papers), Emerging Infectious Diseases (12papers), Proceedings of The National Academy of Sciences of The United States Of America (10 papers) and Asian Journal of Psychiatry (8 papers), Diabetes and Metallic Syndromes Clinical Research and Review (7 papers) and BMJ Clinical Ed (7 papers).

\section{SUMMARY AND CONCLUSION}

The study provides a bibliometric analysis of 103054 publications on COVID-19 disease published during the early stage of the outbreak (January 2020) till February 15, 2021. About 32\% of total publications were financially supported by $1000+$ funding agencies. The USA, China and U.K published the most share of publications (25.56\%, $10.77 \%$ and $10.70 \%$ share respectively), followed by Italy $(9.07 \%)$, India $(6.95 \%)$, etc. Medicine is the top most area of research investigation in COVID-19 disease studies with a $71.44 \%$ share of global output, followed by Biochemistry, Genetics and Molecular Biology (10.61), Social Sciences (9.70\%), Immunology and Microbiology (6.71\%), etc. Nearly $15 \%$ of research output is focused on Adults group affected by COVIDE-19 disease, followed by Middle Aged (10.59\%), Aged (9.86\%), Adolescents (3.62\%) and Children group studies (1.55\%). Treatment studies in COVID-19 research account for a $17.94 \%$ share, followed by clinical studies (11.90\%), complications (7.06\%), epidemiological studies (3.60\%) and pathophysiology (3.55\%). Under clinical studies, Virological studies accounted for the most publications ( $9.58 \%$ share), followed by respiratory studies (6.97\%), molecular studies $(3.11 \%)$, genetics studies $(3.09 \%)$, biological studies (2.85\%), immunological studies $(1.30 \%)$, etc. The study also provides a window to key research countries, organizations and authors and their collaborative linkages. It also identified the key source journals. Of the top 50 organizations, 18 were from USA, 7 from Australia, 6 each from China, Italy and U.K., 4 from France, 2 from Canada and 1 each from Brazil, India, Iran, Australia, Singapore, Iran and Hong Kong. Of the top 50 authors, 10 are from U.K., 8 from Italy, 7 from USA, 5 from India, 3 each from France and Hong Kong, 2 each from Belgium and China and 1 each from Columbia, Iran, Malaysia, Switzerland, Canada, Nepal, Saudi Arabia, Indonesia and Singapore. In conclusion it may be stated that COVID-19 disease research is though global in nature but it is the countries like the USA, UK, and China which dominate the field whereas the other participating countries are just distant cousins in COVID-19 research. The results of this study should be of interest to working scientists, clinicians and policy-makers in improving their understanding of COVID-19 disease and in its management.

\section{CONFLICT OF INTEREST}

The authors declare that there is no conflict of interest.

\section{REFERENCES}

1. COVID-19 Disease. 2019. https://www.who.int/emergencies/diseases/novelcoronavirus-2019/question-and-answers-hub/q-a-detail/coronavirus-diseasecovid-19 (Accessed on 30 March 2021)

2. Origin of SARS-CoV-2. 2020. https://apps.who.int/iris/bitstream/handle/10665/332197/WHO-2019-nCoV-FAQ-Virus_origin-2020.1-eng.pdf

3. Interim Clinical Guidance for Management of Patients with Confirmed Coronavirus Disease (COVID-19). U.S. Centers for Disease Control and Prevention (CDC). 2020. (Accessed on 30 March 2021)

4. Oran DP, Topol EJ. The Proportion of SARS-CoV-2 Infections that are Asymptomatic: A systematic review. Annals of Internal Medicine. 2021. M20-6976. Doi:10.7326/M20-6976. 30 June 2020

5. Transmission of COVID-19. European Centre for Disease Prevention and Control. 2020. Retrieved 6 December 2020. https://www.ecdc.europa.eu/en/covid-19/latest-evidence/transmission

6. Coronavirus disease 2019. https://en.wikipedia.org/wiki/Coronavirus_disease_2019.

7. Verdecchia P, Cavallini C, Spanevello A, Angeli F. The pivotal link between ACE2 deficiency and SARS-CoV-2 infection. European Journal of Internal Medicine. 2020;76:14-20. Doi:10.1016/j.ejim.2020.04.037.

8. Sa'ed HZ, Al-Jabi SW. Mapping the situation of research on coronavirus disease-19 (COVID-19): A preliminary bibliometric analysis during the early stage of the outbreak. BMC Infect Dis. 2020;20:561. https://doi.org/10.1186/s12879 020-05293-z.

9. Chahrour M, Assi S, Bejjani M, Nasrallah AA, Salhab H, Fares M, et al. A Bibliometric analysis of COVID-19 research activity: A call for increased output. Cureus. 2020;12(3):e7357.

10. Diéguez-Campa CE, Pérez-Neri I, Reyes-Terán G, Flores-Apodaca IA, CastilloLedón-Pretelini J, Mercado-Bautista O, et al. The 2020 research pandemic: A bibliometric analysis of publications on COVID-19 and their scientific impact during the first months. Arch Cardiol Mex. 2020. Doi: 10.24875/ACM.20000370.

11. Yu Y, Li Y, Zhang Z, Gu Z, Zhong H, Zha Q, Yang L, Zhu C, Chen E. A bibliometric analysis using VOSviewer of publications on COVID-19. Ann Transl Med. 2020;8(13):816. Doi: 10.21037/atm-20-4235. PMID: 32793661.

12. Haghani M, Bliemer MC, Goerlandt F, Li J. The scientific literature on coronaviruses, COVID-19 and its associated safety-related research dimensions: A scientometric analysis and scoping review. Saf Sci. 2020;129:104806. doi: 10.1016/j.ssci.2020.104806. Epub 2020 May 7

13. Lou J, Tian SJ, Niu SM, Kang XQ, Lian HX, Zhang LX, et al. Coronavirus disease 2019: A bibliometric analysis and review. Eur Rev Med Pharmacol Sci. 2020;4(6):3411-21. DOI: 10.26355/eurrev_202003_20712

14. Mao X, Guo L, Fu P, Xiang C. The status and trends of coronavirus research: A global bibliometric and visualized analysis. Medicine. 2020;99(22):e20137. doi: 10.1097/MD.0000000000020137.

15. Tao Z, Zhou S, Yao R, Wen K, Da W, Meng Y, et al. COVID-19 will stimulate a new coronavirus research breakthrough: A 20-year bibliometric analysis. Ann Trans Med. 2020;8(8):528. https://atm.amegroups.com/article/view/40214/html

16. Zhai $F$, Zhai $Y$, Cong $C$, Song $T$, Xiang R, Feng $T$, et al. Research Progress of Coronavirus Based on Bibliometric Analysis. Int J Environ Res Public Health. 2020;17(11):3766. doi: 10.3390/ijerph17113766

17. Zhou Y, Chen L. Twenty-Year Span of Global Coronavirus Research Trends: A Bibliometric Analysis. Int J Environ Res Public Health 2020. 2020;17(9):3082. https://doi.org/10.3390/ijerph17093082 